\title{
Supplementary Materials to the manuscript
}

The Reaction of the $\mathrm{Si}_{8} \mathrm{O}_{20}\left(\mathrm{SnMe}_{3}\right)_{8}$ Building Block with Silyl Chlorides: A New Synthetic Methodology for Preparing Nanostructured Building Block Solids

\author{
Jason C. Clark, Craig E. Barnes*
}

Department of Chemistry, University of Tennessee, Knoxville, TN 37996-1600

Submitted to Chemistry of Materials

Table of Contents:

Figure $\mathrm{S} 1$

${ }^{29} \mathrm{Si}$ Solid state NMR (SSNMR) spectra (MAS) of the product from the reaction of $\mathrm{HSiCl}_{3}$ with $\mathrm{Si}_{8} \mathrm{O}_{20}\left(\mathrm{SnMe}_{3}\right)_{8}$ in hexane with varying initial stoichiometries of $\mathrm{HSiCl}_{3}: \mathrm{Si}_{8} \mathrm{O}_{20}\left(\mathrm{SnMe}_{3}\right)_{8}$

Figure $\mathrm{S} 2$

${ }^{29} \mathrm{Si}$ Solid state NMR (SSNMR) spectra (MAS) of the product from the reaction of $\mathrm{Me}_{2} \mathrm{SiCl}_{2}$ with $\mathrm{Si}_{8} \mathrm{O}_{20}\left(\mathrm{SnMe}_{3}\right)_{8}$ in toluene with varying initial stoichiometries of $\mathrm{Me}_{2} \mathrm{SiCl}_{2}: \mathrm{Si}_{8} \mathrm{O}_{20}\left(\mathrm{SnMe}_{3}\right)_{8}$

Figure S3

${ }^{29} \mathrm{Si}$ Solid state NMR (SSNMR) spectra (MAS) of the product from the reaction of $\mathrm{Me}_{2} \mathrm{SiCl}_{2}$ with $\mathrm{Si}_{8} \mathrm{O}_{20}\left(\mathrm{SnMe}_{3}\right)_{8}$ in hexane with varying initial stoichiometries of $\mathrm{Me}_{2} \mathrm{SiCl}_{2}: \mathrm{Si}_{8} \mathrm{O}_{20}\left(\mathrm{SnMe}_{3}\right)_{8}$

Figure S4

$\mathrm{BJH}$ pore volume distribution for the reaction of $\mathrm{HSiCl}_{3}$ and $\mathrm{Si}_{8} \mathrm{O}_{20}\left(\mathrm{SnMe}_{3}\right)_{8}$ in toluene This pore size distribution is typical of all the high surface area solids investigated in these studies.

Figure S5

${ }^{29} \mathrm{Si}$ SSNMR (MAS and CPMAS) of a nanostructure solid containing only "embedded" $\mathrm{Me}_{2} \mathrm{Si}(\mathrm{OSi} \equiv)_{2}$ groups linking $\mathrm{Si}_{8} \mathrm{O}_{20}$ building blocks. $\mathrm{SiCl}_{4}$-derived linking groups are also present in the matrix.

Figure S6

${ }^{29} \mathrm{Si}$ SSNMR (MAS and CPMAS) of a nanostructure solid containing only "surface" $\mathrm{Me}_{2} \mathrm{SiCl}(\mathrm{OSi} \equiv)$ groups linking $\mathrm{Si}_{8} \mathrm{O}_{20}$ building blocks. $\mathrm{SiCl}_{4}$-derived linking groups are also present in the matrix.

Table S1

BET Surface area analysis of the solids resulting from the reaction of $\mathrm{Me}_{2} \mathrm{SiCl}_{2}$ with $\mathrm{Si}_{8} \mathrm{O}_{20}\left(\mathrm{SnMe}_{3}\right)_{8}$ under the conditions given. Absorption gas: nitrogen. Pore size distributions calculated using standard BJH equations. 
Table S2

BET Surface area analysis of the solids resulting from the reaction of $\mathrm{HSiCl}_{3}$ with $\mathrm{Si}_{8} \mathrm{O}_{20}\left(\mathrm{SnMe}_{3}\right)_{8}$ under the conditions given. Absorption gas: nitrogen. Pore size distributions calculated using standard BJH equations.

Figure S1

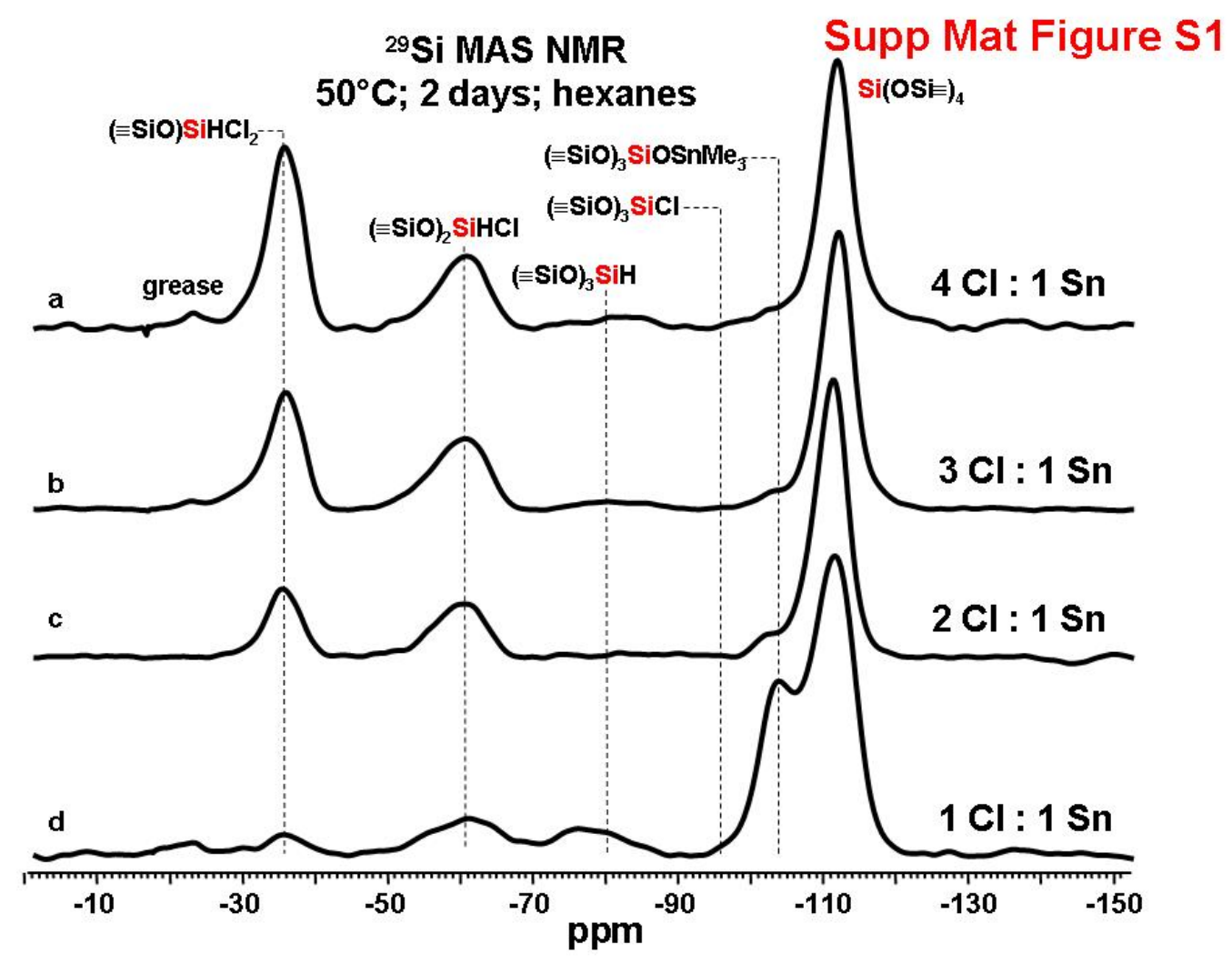

Figure S2

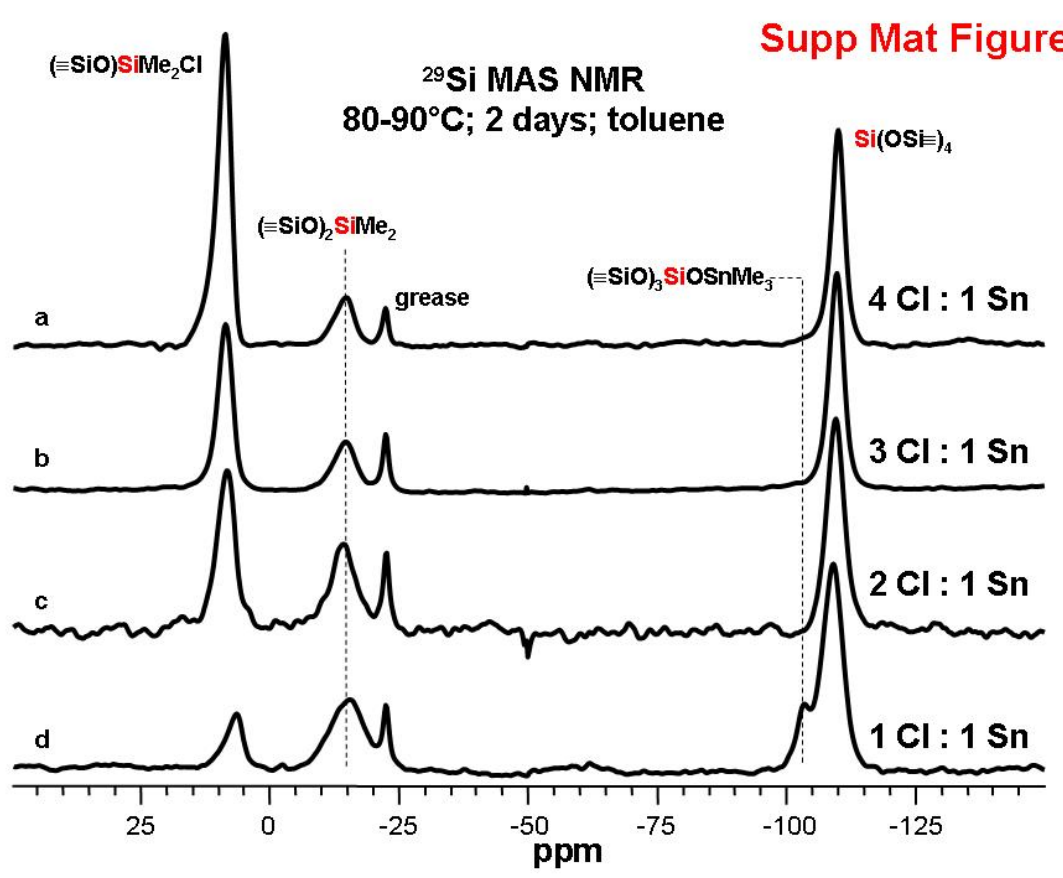


Figure S3

${ }^{29}$ Si MAS NMR

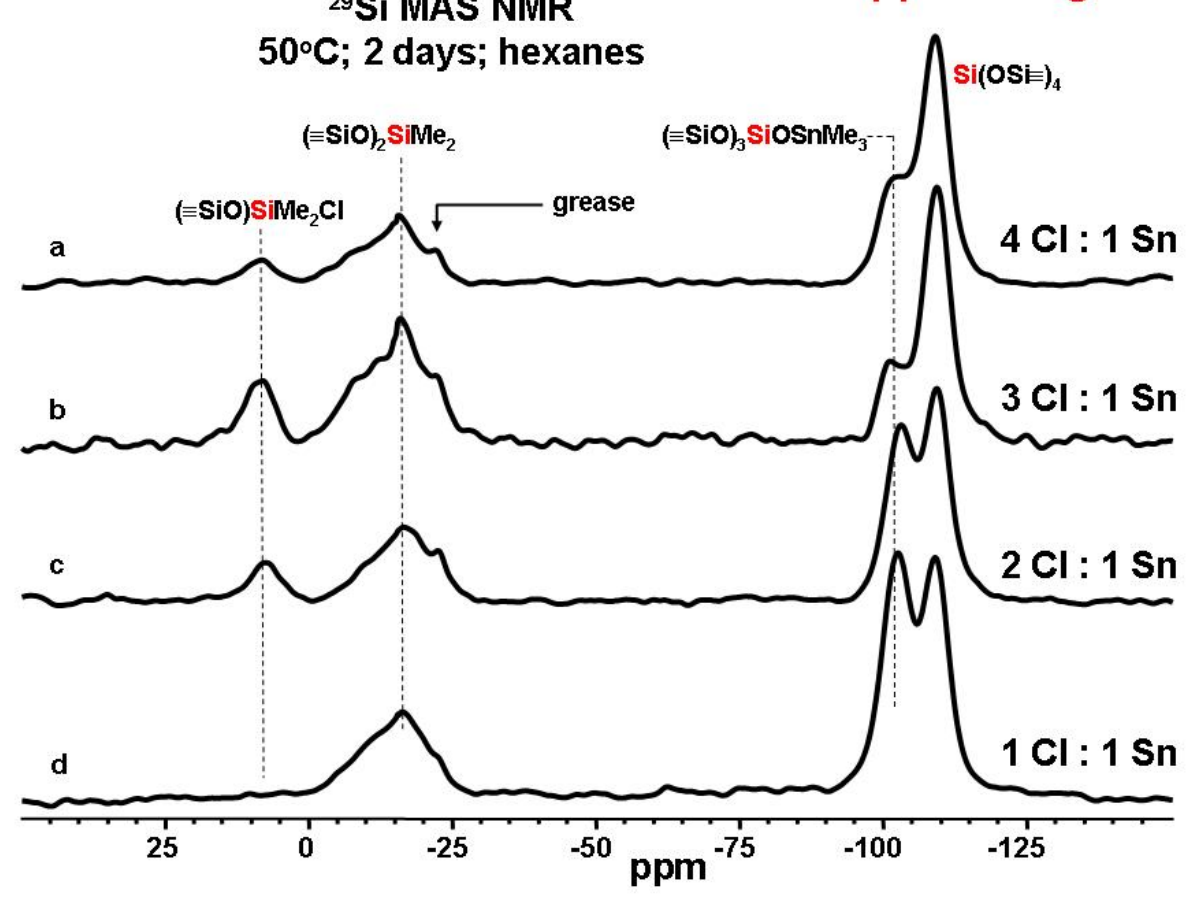

Figure S4

BJH pore volume distribution for the reaction of $\mathrm{HSiCl}_{3}$ Supp Mat Figure S4 and $\mathrm{Si}_{8} \mathrm{O}_{20}\left(\mathrm{SnMe}_{3}\right)_{8}$ in toluene

Total surface area: $672 \mathrm{~m}^{2} / \mathrm{g}$

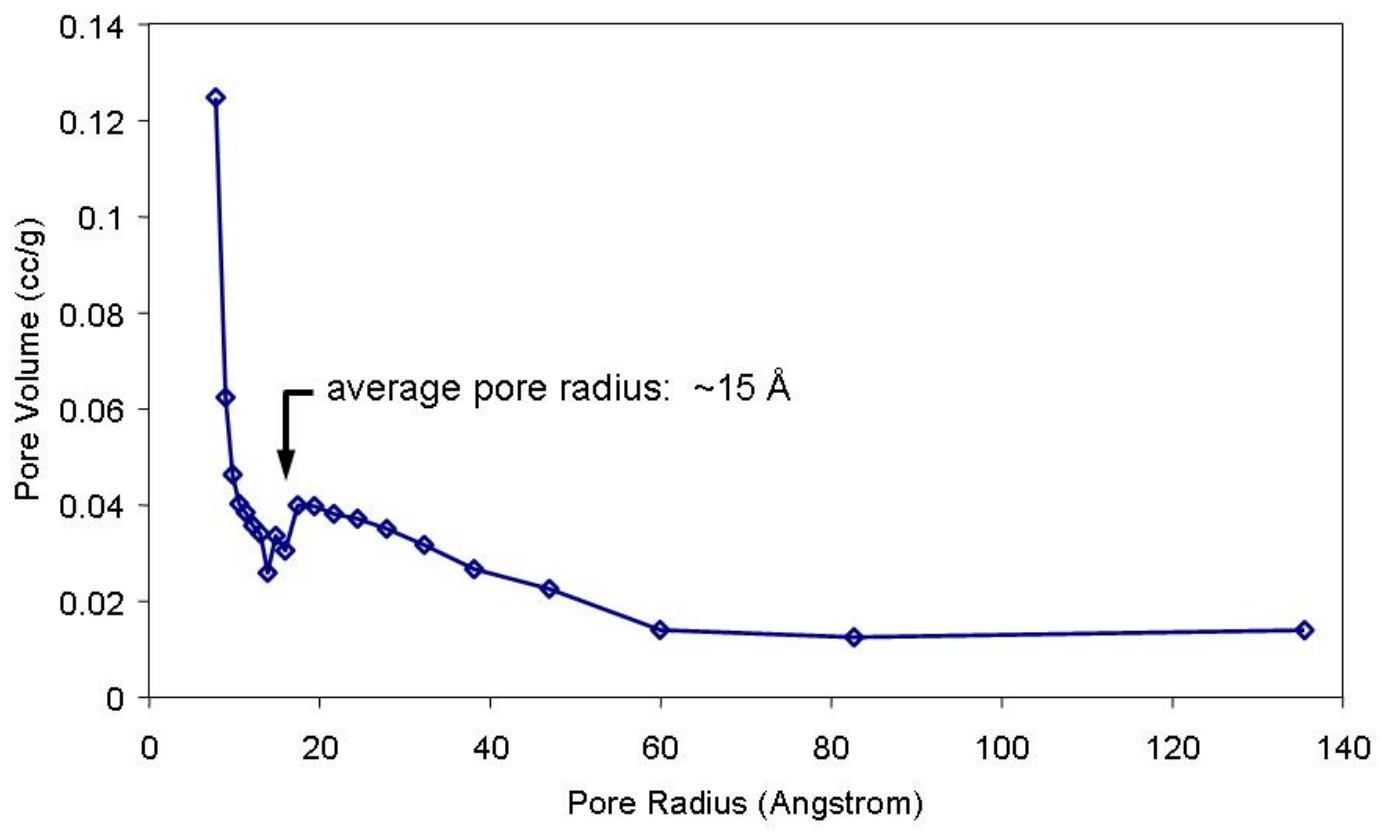


Figure S5

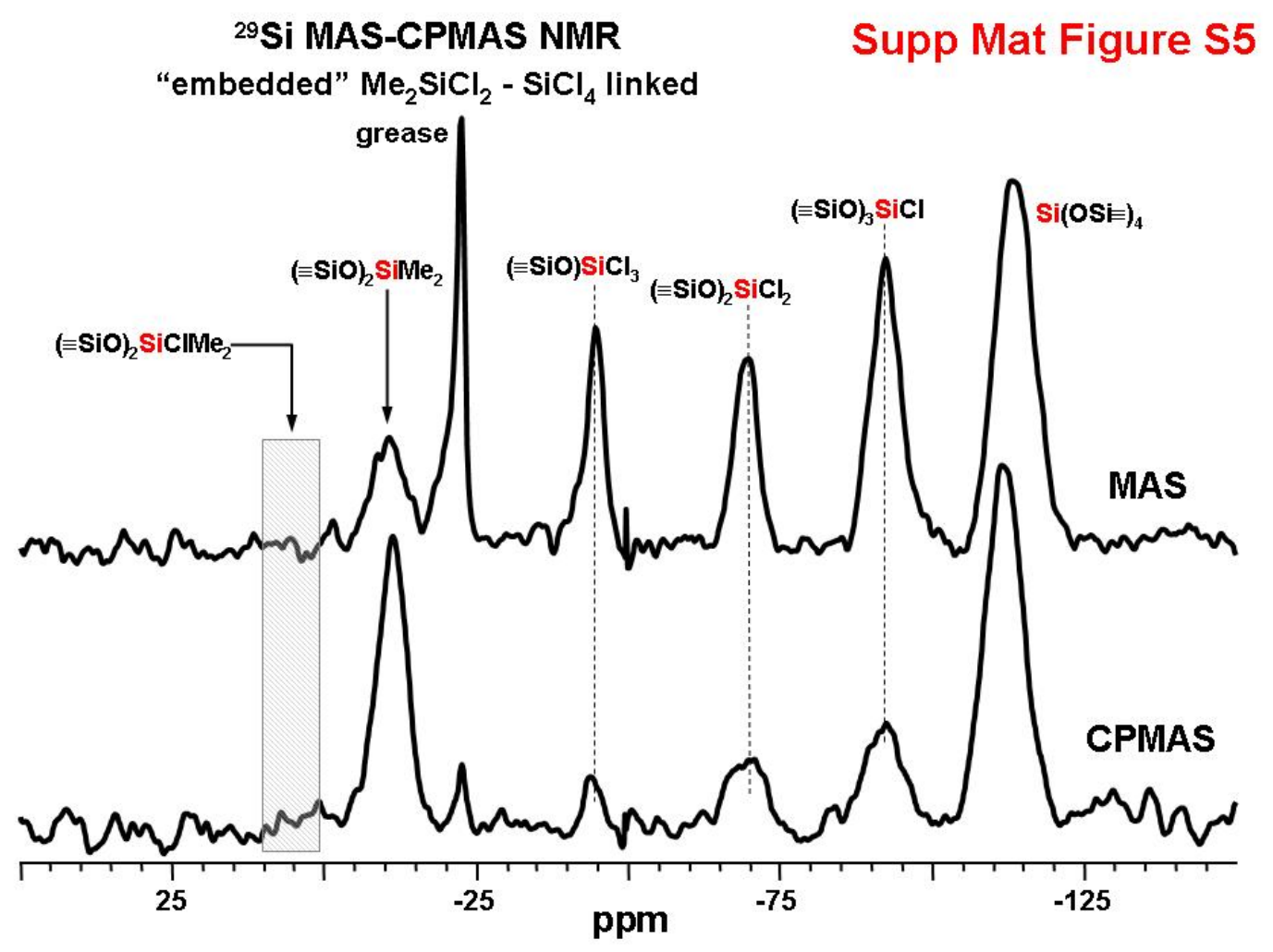

Figure S6

${ }^{29}$ Si MAS-CPMAS NMR

"capping" $\mathrm{Me}_{2} \mathrm{SiCl}-\mathrm{SiCl}_{4}$ cross linked

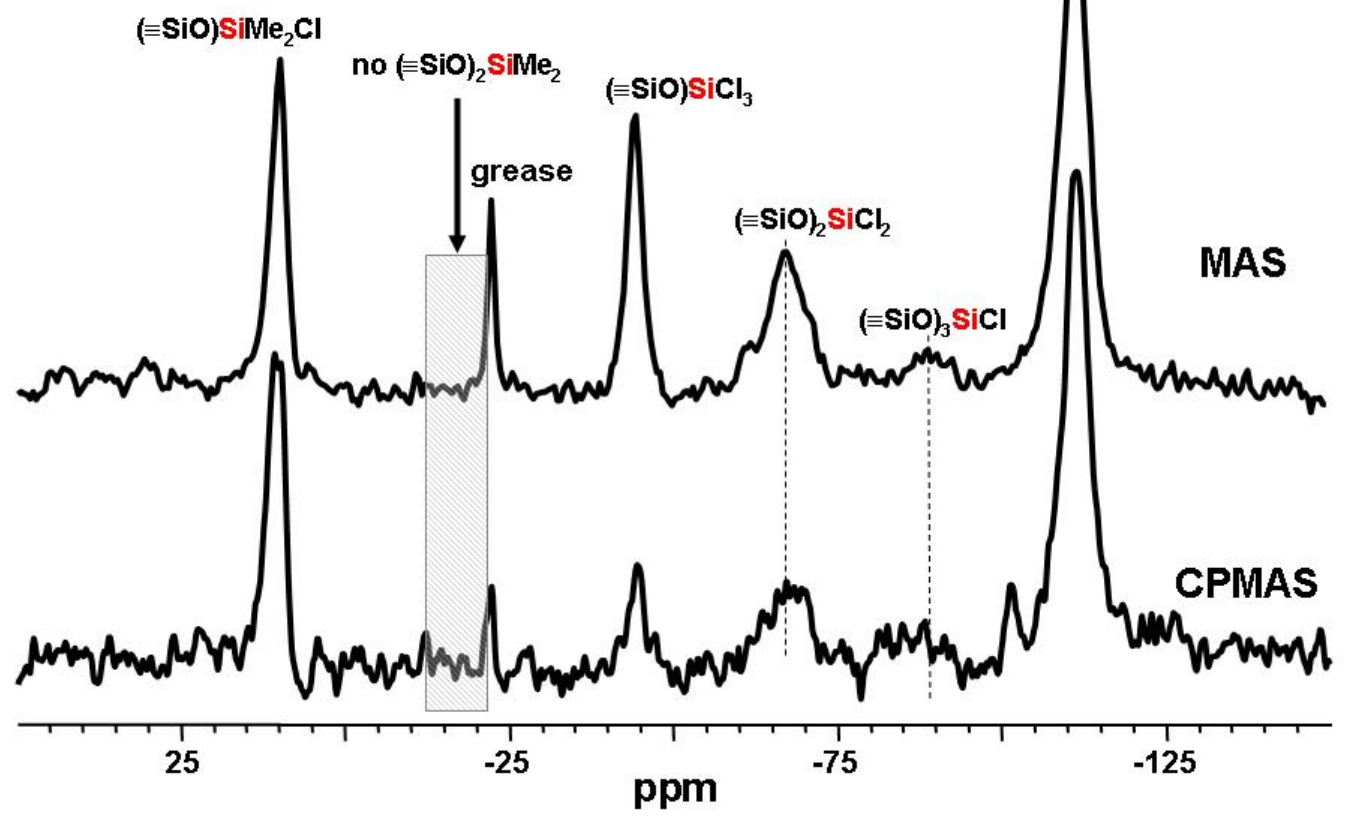

Supp Mat Figure S6 $(\equiv \mathrm{SiO})_{4} \mathrm{Si}$<smiles>[Mg]</smiles> 
Table S1

\section{Supp Mat Table S1}

Table S1 BET Surface area analysis of the solids resulting from the reaction of $\mathrm{Me}_{2} \mathrm{SiCl}_{2}+\mathrm{Si}_{8} \mathrm{O}_{20}\left(\mathrm{SnMe}_{3}\right)_{8}$ under the conditions given. Absorption gas: nitrogen. Pore size distributions calculated using standard BJH equations.

\begin{tabular}{|c|c|c|c|c|}
\hline Conditions & Cl:Sn & $\begin{array}{c}\text { Surface } \\
\text { Area }\left(\mathrm{m}^{2} / \mathrm{g}\right)\end{array}$ & $\begin{array}{c}\text { Total Pore } \\
\text { Volume }(\mathrm{cc} / \mathrm{g})\end{array}$ & $\begin{array}{c}\text { Average Pore } \\
\text { Radius }(\AA)\end{array}$ \\
\hline Toluene, $80-90^{\circ} \mathrm{C}, 2$ days & $4: 1$ & low & low & low \\
\hline Toluene, $80-90^{\circ} \mathrm{C}, 2$ days & $3: 1$ & low & low & low \\
\hline Toluene, $80-90^{\circ} \mathrm{C}, 2$ days & $2: 1$ & 172 & 0.150 & 17 \\
\hline Toluene, $80-90^{\circ} \mathrm{C}, 2$ days & $1: 1$ & 669 & 0.496 & 15 \\
\hline Hexanes, $50^{\circ} \mathrm{C}$, overnight & $4: 1$ & 13 & 0.015 & 24 \\
\hline Hexanes, $50^{\circ} \mathrm{C}$, overnight & $3: 1$ & 29 & 0.171 & 13 \\
\hline Hexanes, $50^{\circ} \mathrm{C}$, overnight & $2: 1$ & 12 & 0.010 & 19 \\
\hline Hexanes, $50^{\circ} \mathrm{C}$, overnight & $1: 1$ & 14 & 0.021 & 30 \\
\hline
\end{tabular}

Table S2

\section{Supp Mat Table S2}

Table S2 BET Surface Area Analysis of the solids resulting from the reaction of $\mathrm{HSiCl}_{3}+\mathrm{Si}_{8} \mathrm{O}_{20}\left(\mathrm{SnMe}_{3}\right)_{8}$ under the conditions given.

Absorption gas: nitrogen. Pore size distributions calculated using standard BJH equations.

\begin{tabular}{|c|c|c|c|c|}
\hline Conditions & Cl:Sn & $\begin{array}{c}\text { Surface } \\
\text { Area }\left(\mathrm{m}^{2} / \mathrm{g}\right)\end{array}$ & $\begin{array}{c}\text { Total Pore } \\
\text { Volume }(\mathrm{cc} / \mathrm{g})\end{array}$ & $\begin{array}{c}\text { Average Pore } \\
\text { Radius (A) }\end{array}$ \\
\hline Toluene, $80-90^{\circ} \mathrm{C}, 2$ days & $4: 1$ & low & low & low \\
\hline Toluene, $80-90^{\circ} \mathrm{C}, 2$ days & $3: 1$ & 558 & 0.400 & 14 \\
\hline Toluene, $80-90^{\circ} \mathrm{C}, 2$ days & $2: 1$ & 660 & 0.513 & 16 \\
\hline Toluene, $80-90^{\circ} \mathrm{C}, 2$ days & $1: 1$ & 672 & 0.539 & 16 \\
\hline Hexanes, $50^{\circ} \mathrm{C}$, overnight & $4: 1$ & 336 & 0.243 & 14 \\
\hline Hexanes, $50^{\circ} \mathrm{C}$, overnight & $3: 1$ & 324 & 0.254 & 16 \\
\hline Hexanes, $50^{\circ} \mathrm{C}$, overnight & $2: 1$ & 401 & 0.295 & 15 \\
\hline Hexanes, $50^{\circ} \mathrm{C}$, overnight & $1: 1$ & 58 & 0.046 & 16 \\
\hline
\end{tabular}

